\title{
Thrombokinetics in Man
}

\author{
Laurence A. Harker and Clement A. Finch \\ From the Department of Medicine, University of Washington School of \\ Medicine, Seattle, Washington 98105
}

A B S T R A C T Platelet production, distribution, and destruction have been quantitated in normal man and in selected patients with platelet disorders. In most instances, total production as calculated from the megakaryocyte mass agreed with production estimated from platelet turnover. In patients with megaloblastosis, a discrepancy between these two measurements indicated the presence of ineffective thrombopoiesis.

Thrombopoiesis was regulated by $(a)$ alterations in megakaryocyte number, and $(b)$ changes in megakaryocyte volume (produced by changes in endomitosis). The volume-endomitosis changes were closely related to the peripheral platelet count and were a useful indicator of thrombopoietic stimulus.

Thrombocytopenic disorders have been classified on the basis of the disturbed physiology into disorders of (a) production (hypoproliferative or ineffective), (b) distribution (splenic pooling), or (c) destruction (immune or consumptive). Less than a twofold increase in platelet production in the presence of significant thrombocytopenia was taken to represent impaired proliferation.

Thrombocytosis was classified as reactive or autonomous. Reactive thrombocytosis was consistently associated with a mean megakaryocyte volume and endomitosis less than normal but appropriate for the elevated circulating platelet count. In contrast, the average megakaryocyte volume and nuclear number were always greater than normal in thrombocythemia findings indicating autonomy.

\section{INTRODUCTION}

During the past $20 \mathrm{yr}$, studies on the production, survival, and destruction of red cells have permitted a precise kinetic classification of red cell disorders. Similar studies on platelets have been limited by difficulties in the

Dr. Harker is Assistant Professor of Medicine, University of Washington. Dr. Finch is Professor and Chief, Division of Hematology, University of Washington.

Received for publication 5 August 1968 and in revised form 21 February 1969. methodology for accurate quantitative measurement. This paper describes the procedures developed in this laboratory for measuring platelet production and turnover in the circulation and presents a physiological classification of platelet disorders derived from measurements in human subjects.

\section{METHODS}

Measurements have been made of megakaryocyte mass, platelet count, distribution, and survival in 15 normal subjects and in 68 patients with platelet abnormalities. ${ }^{1}$

Platelet counts were performed with an electronic particle counter $^{2}$ on peripheral blood collected in ethylenediaminetetraacetate (EDTA) according to the method of Bull, Schneiderman, and Brecher (1), in which the red cells are separated from the platelet-containing plasma by gravity sedimentation in plastic tubes. The blood platelet count is derived from the plasma platelet count by using the hematocrit correction factor. Since settling of platelets occurred in samples standing longer than $20-30 \mathrm{~min}$, such samples were remixed by inverting and reinverting the sedimenting tube immediately before the plasma was sampled. This procedure mixed the platelets in the supernatant plasma but did not greatly disturb the plasma red cell interface. Dilution was varied according to the degree of thrombocytopenia to ensure a counting rate significantly above background. The mean platelet count in 98 normal subjects performed with the electronic particle counter was 250,000 plateletes $/ \mu 1 \pm 43,000$ (1 SD). The correlation observed between platelet counts performed with the electronic particle counter and phase microscopy (2) is shown in Fig. 1.

Platelet survival was determined from the isotope disappearance curve after the injection of ${ }^{51} \mathrm{Cr}$-labeled platelets by use of the acid-citrate method (3). This technic involved the separation of platelet-rich plasma from acid citrate dextrose (ACD)-drawn blood by differential centrifugation and the subsequent adjustment of plasma $\mathrm{pH}$ to 6.5 with $0.15 \mathrm{M}$ citric acid. Platelets were removed from plasma by centrifugation, and a concentrated platelet suspension was produced by gently resuspending the resultant platelet button in a small amount of residual plasma. The platelet suspension

\footnotetext{
1 The pertinent clinical data are available by requesting Document No. 00354 from the American Documentation Institute, National Auxiliary Publication Service of the American Society for Information Science, c/o CCM Information Science, Inc., 22 West 34th St., New York 10001.

${ }^{2}$ Model B, $50 \mu$ aperture tube, Coulter Electronics, Inc., Hialeah, Fla.
} 
was incubated for at least 20 min with approximately $300 \mu \mathrm{C}$ of ${ }^{5} \mathrm{Cr}$ (as chromate in isotonic $\mathrm{NaCl}$ ). The ${ }^{5} \mathrm{Cr}$-labeled platelets were separated from the unbound chromium by centrifugation and subsequent careful washing of the platelet button with $30 \mathrm{ml}$ of platelet-poor plasma. After resuspension of the platelets in platelet-poor plasma and preparation of a standard, the remaining ${ }^{51} \mathrm{Cr}$-labeled platelets were quantitatively injected into the patient. The entire procedure was carried out under sterile conditions at room temperature using plastic equipment. ${ }^{8}$ In subjects with platelet counts greater than 25,000 platelets/ $\mu 1$, platelet survival was determined using autologous platelets. In patients with more severe thrombocytopenia, homologous-labeled platelets from ABO compatible, hepatitis-free donors were transfused. With two exceptions (patient Nos. 17 and 22), the subjects studied with labeled donor platelets had not previously received blood transfusions. Platelet survival was estimated directly from linear disappearance curves by extrapolating to zero activity. When an exponential disappearance curve was obtained, survival was calculated from the half-time divided by the natural logarithm of 2 (4). For disappearance curves falling between linear and exponential patterns, survival was estimated from extrapolation of the initial slope to zero activity as described by Mills (5).

The proportion of labeled platelets remaining in the systemic circulation after infusion, i.e. "recovery," was calculated from the platelet activity per milliliter extrapolated to zero time, multiplied by the estimated blood volume, and divided by the platelet ${ }^{-1} \mathrm{Cr}$ activity injected. The blood volume was estimated from height and weight measurements (6).

Platelet turnover per microliter per day was calculated from the peripheral platelet count divided by the platelet survival time in days and corrected for recovery. Expected recovery as found in splenectomized patients was $90 \%$, and any smaller value was assumed to be due to splenic pooling (7). The recovery factor to correct platelet turnover for sequestration in the spleen was

$$
\frac{90 \%}{\% \text { recovery in the patient }}
$$

The number of megakaryocytes in the marrow was determined by relating megakaryocytes to nucleated red cells and then estimating erythroid mass from plasma iron turnover measurements $(8,9)$ using the formula:

$$
M=\left(\frac{M_{0} R_{0}}{P_{0}}\right)\left(\frac{P}{R}\right)
$$

The megakaryocyte: erythroid ratio used in this formula was determined from bone marrow biopsies which were obtained with a $10 \mathrm{~g}$ Silverman needle, ${ }^{5}$ fixed in Zenker's formaldehyde, and subsequently decalcified with $5 \%$ formic acid for $24 \mathrm{hr}$. After paraffin embedding, the sections were cut at $3 \mu$ thickness and placed overnight in Giemsa stain followed by decolorization with $95 \%$ ethanol. The ratio of

${ }^{8}$ Fenwal Laboratories, Morton Grove, Ill.

${ }^{4} \mathrm{R}=\mathrm{E} / \mathrm{M} ; \mathrm{E} \propto \mathrm{P} ; \mathrm{M} \propto \mathrm{P} / \mathrm{R} ; \mathrm{M} / \mathrm{M}_{0}=\mathrm{P} \cdot \mathrm{R}_{0} / \mathrm{R} \cdot \mathrm{P}_{0} ;$ $M=M_{0} R_{0} / P_{0} P / R$, where: $R=$ ratio of nucleated erythroid cells to megakaryocytes; $\mathrm{E}=$ number of nucleated erythroid cells; $M=$ number of megakaryocytes; $P=$ plasma iron turnover measurement; subscript " 0 " refers to normal ; $\mathrm{M}_{0}=6.1 \times 10^{6} / \mathrm{kg} ; \mathrm{R}_{0}=465: 1 ; \mathrm{P}_{0}=\mathrm{mg} \mathrm{Fe} / 100 \mathrm{ml} \mathrm{WB}$ per $24 \mathrm{hr}$, (0.70 mg \pm 0.09 male, $0.60 \mathrm{mg} \pm 0.10$ female).

${ }^{5}$ Westerman-Jensen marrow biopsy needle, Becton-Dickinson \& Co., Rutherford, N. J.

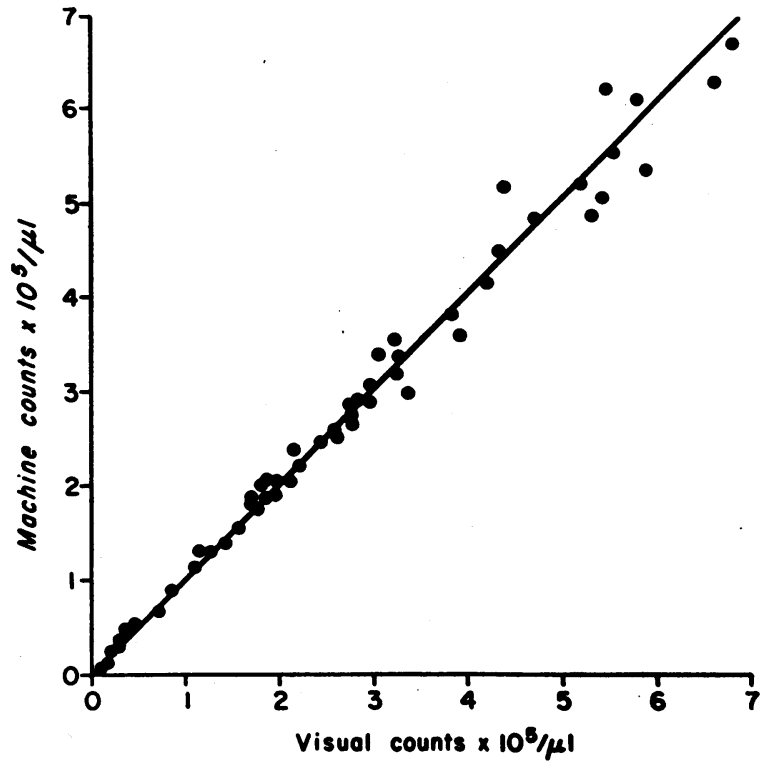

FIGURE 1 A comparison of platelet counting performed by machine (Coulter counter) and visual (phase microscopy) methods $(1,2)$.

nucleated red cells to megakaryocytes was determined by counting at least 20,000 nucleated red cells and the corresponding megakaryocytes in bone marrow sections. Because the thickness of megakaryocytes was greater than the section thickness, the ratio was corrected for the multiple counting factor (i.e., the average number of sections encompassing megakaryocyte thickness) (8). In the normal subject this figure was determined to be 6.7 (normal mean megakaryocyte diameter $20.8 \mu \pm 3.3$ ). In the pathological states, the average megakaryocyte diameter varied widely. Accordingly, the corresponding multiple counting factor was determined from serial sections of 100 megakaryocytes with diameters extending over the range of $13-35 \mu$. The average multiple counting factor was found to approximate closely the mean megakaryocyte diameter of the patient divided by 3.10.

The number of nucleated erythroid cells in the marrow was more directly determined in five subjects with polycythemia vera by the isotope dilution technic adapted for tissue section, which required approximately $1 \mathrm{mc}$ of ${ }^{\circ} \mathrm{Fe}$ for each study (8). In this method the number of erythroid cells was calculated by dividing the radioactivity in a marrow section by the number of erythroid cells in the same section and relating this ratio to the total amount of ${ }^{60} \mathrm{Fe}$ activity in the marrow at the time of sampling. Since the amount of radioiron localized in the erythroid marrow of man at the time of sampling could not be directly measured, an assumed value of $67 \%$ was used as previously determined from animal studies (10).

A straight-line relationship was seen between plasma iron turnover and erythroid number in these five patients (Fig. 2). Based on these results and further studies on normal subjects and a large number of patients (9-12) the plasma iron turnover was assumed to be an acceptable measurement of erythroid mass.

The mean megakaryocyte diameter was determined from planimetric measurements of at least 50 nucleus-containing megakaryocyte cuts on random-section photomicrographic 


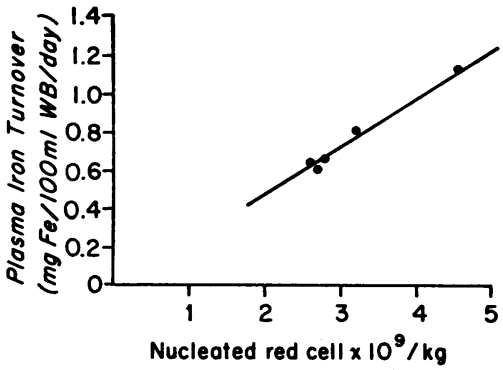

FIgURE 2 The relationship between plasma iron turnover and nucleated red cell number as determined in five patients by the marrow section, isotope dilution method (8).

enlargements (8) for each subject. When a spheroidal shape for megakaryocytes in sectioned material is assumed, the mean megakaryocyte volume was calculated from the diameter measurements. Megakaryocyte mass was the product of the mean megakaryocyte volume and the total megakaryocyte number (13).

The megakaryocyte nuclear lobe number of at least 100 megakaryocytes from each subject was counted under oil immersion, phase-microscopy in acetocarmine-fixed marrow squash preparation (14). On the basis of the counted number of nuclear lobes, the megakaryocytes were classified as containing $2,4,8,16$, or 32 nuclei. When the number of nuclear lobes fell between these values, the cell was grouped with the next highest class. The cell volume per nuclear lobe, i.e. nuclear unit, was calculated by dividing the mean megakaryocyte volume by the average nuclear number (13).
The daily platelet production per megakaryocyte nuclear unit was determined by dividing the daily platelet turnover per kilogram of body weight (i.e. platelet turnover per microliter per day $\times$ blood volume per kilogram) by the number of nuclear units per kilogram (i.e., megakaryocyte mass/kilogram per cell volume per nucleus). This value is expressed as platelets per nuclear unit per day.

Platelet turnover reflects platelet removal from the circulation. In the stable state, however, the platelet turnover is also a measure of platelet release from the marrow into the circulation and will be referred to as effective platelet production. Since megakaryocyte mass reflects the plateletproducing capacity of the marrow, this measurement will be referred to as total platelet production (13). Significant disparity between the productive activity of the marrow (megakaryocyte mass) and the effective delivery of the platelets into the circulation (platelet turnover) is believed to represent ineffective thrombopoiesis.

\section{RESULTS}

\section{Normal thrombokinetics}

The results of thrombokinetic measurements in 15 normal subjects are shown in Table I. They had a mean platelet count of 250,000 platelets $/ \mu 1 \pm 35,000$, $^{\circ}$ immediate recoveries in the systemic circulation of injected ${ }^{51} \mathrm{Cr}$ labeled platelets of $64.6 \% \pm 4.1$, and a mean platelet survival of 9.9 days \pm 0.6 (Fig. 3). The platelet- ${ }^{\text {"1 }} \mathrm{Cr}-$ bound activity not in the circulation was presumed to

- This and subsequent variances are all \pm 1 SD.

TABLE I

Normal Thrombokinetics

\begin{tabular}{|c|c|c|c|c|c|c|c|c|c|c|c|}
\hline \multirow[b]{3}{*}{$\begin{array}{c}\text { Subject } \\
\text { No. }\end{array}$} & \multirow{2}{*}{\multicolumn{5}{|c|}{ Effective thrombopoiesis }} & \multicolumn{4}{|c|}{ Total thrombopoiesis } & \multirow[b]{3}{*}{$\begin{array}{c}\text { Cell } \\
\text { volume/ } \\
\text { nucleus }\end{array}$} & \multirow[b]{3}{*}{$\begin{array}{l}\text { Platelet } \\
\text { production/ } \\
\text { nuclear } \\
\text { unit }\end{array}$} \\
\hline & & & & & & \multirow[b]{2}{*}{ Number } & \multicolumn{2}{|c|}{ Megakaryocyte } & \multirow[b]{2}{*}{$\begin{array}{l}\text { Total } \\
\text { produc- } \\
\text { tion }\end{array}$} & & \\
\hline & Count & Survival & Recovery & Turnover & $\begin{array}{l}\text { Effec- } \\
\text { tive pro- } \\
\text { duction }\end{array}$ & & Volume* & Mass & & & \\
\hline & $\mu l$ & days & $\%$ & $\begin{array}{l}\text { platelets/ } \\
\text { ml per day }\end{array}$ & Xnormal & $\times 10^{\circ} / \mathrm{kg}$ & $\mu^{8}$ & $\begin{array}{c}\mu^{3} \times 10^{10} \\
k g\end{array}$ & Xnormal & $\mu^{3}$ & $\begin{array}{l}\text { platelets/ } \\
N U \text { per } \\
\text { day }\end{array}$ \\
\hline 1 & 231,000 & 9.6 & 62.3 & 34,800 & 1.0 & 5.8 & 4.650 & 2.7 & 1.0 & 505 & 49 \\
\hline 2 & 204,000 & 10.2 & 55.9 & 32,200 & 0.9 & 5.1 & 4.800 & 2.4 & 0.9 & 495 & 50 \\
\hline 3 & 230,000 & 10.4 & 65.8 & 30,200 & 0.9 & 6.5 & 4,750 & 3.1 & 1.1 & 515 & 38 \\
\hline 4 & 267,000 & 9.9 & 68.0 & 35,700 & 1.0 & 5.4 & 4,700 & 2.5 & 0.9 & 532 & 57 \\
\hline 5 & 289,000 & 9.8 & 69.2 & 38,400 & 1.1 & 6.3 & 4,700 & 3.0 & 1.1 & 514 & 49 \\
\hline 6 & 240,000 & 9.0 & 59.0 & 40.700 & 1.1 & 6.2 & 4,700 & 2.9 & 1.0 & & 55 \\
\hline 7 & 260,000 & 10.3 & 69.0 & 33,000 & 0.9 & 7.0 & 4,500 & 3.2 & 1.1 & 500 & 39 \\
\hline 8 & 284,000 & 8.8 & 67.9 & 42,800 & 1.2 & 6.1 & 4,650 & 2.8 & 1.0 & 502 & 57 \\
\hline 9 & 252,000 & 10.2 & 65.0 & 34,200 & 1.0 & 6.8 & 4,700 & 3.2 & 1.1 & & 42 \\
\hline 10 & 240,000 & 9.6 & 68.0 & 33,100 & 1.0 & 6.2 & 4,700 & 2.9 & 1.0 & 533 & 46 \\
\hline 11 & 288,000 & 9.9 & 62.3 & 42,000 & 1.2 & 6.9 & 4,650 & 3.2 & 1.1 & 523 & 51 \\
\hline 12 & 192,000 & 9.6 & 60.0 & 30,000 & 0.9 & 5.0 & 4,900 & 2.5 & 0.9 & 549 & 49 \\
\hline 13 & 215,000 & 10.2 & 63.8 & 29,800 & 0.8 & 6.9 & 4,600 & 3.2 & 1.1 & 503 & 46 \\
\hline 14 & 319,000 & 10.5 & 69.5 & 39,300 & 1.1 & 5.5 & 4,650 & 2.6 & 0.9 & 540 & 51 \\
\hline 15 & 246,000 & 9.8 & 63.2 & 35,800 & 1.0 & 5.9 & 4,650 & 2.7 & 1.0 & 511 & 51 \\
\hline \multirow[t]{2}{*}{ Mean } & 250,000 & 9.9 & 64.6 & 35,000 & 1.0 & 6.1 & 4,700 & 2.8 & 1.0 & 520 & 49 \\
\hline & $\pm 35,000$ & \pm 0.6 & \pm 04.1 & $\pm 4,300$ & \pm 0.1 & \pm 0.7 & \pm 100 & \pm 0.3 & \pm 0.1 & \pm 20 & \pm 5 \\
\hline
\end{tabular}

* Because of shrinkage due to Zenker's formaldehyde fixation, these values do not represent absolute estimates. Diameter measurements from osmic acidfixed samples were $25 \%$ greater. 


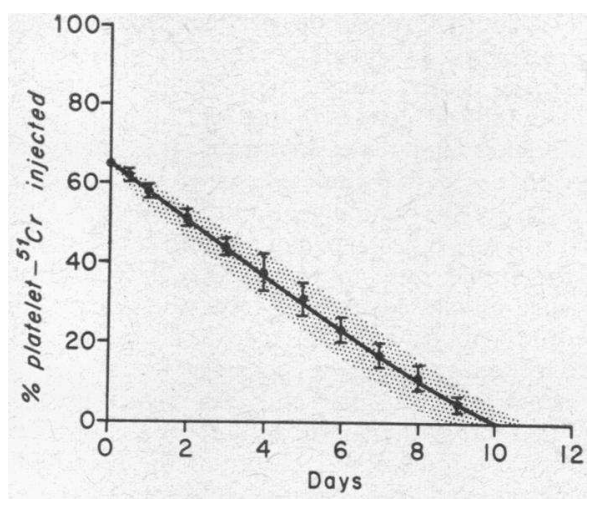

FIGURE 3 A composite platelet disappearance curve based on studies in 15 normal subjects. The immediate recovery was $64.6 \% \pm 4.1$ and was determined by extrapolating the platelet- ${ }^{51} \mathrm{Cr}$ activity in circulation to zero time and dividing this value by the platelet- ${ }^{51} \mathrm{Cr}$ activity injected. In order to depict platelet disappearance only, the zero time platelet $-{ }^{51} \mathrm{Cr}$ activities of the subjects were equated. The solid bar represents $1 \mathrm{SD}$ from the mean, and the range of values is indicated by the shaded area.

reflect largely the splenic platelet pool inasmuch as the recovery in 10 subjects after splenectomy was $90.2 \%$ \pm 9.0 . The platelet turnover in normal subjects was

$$
\frac{250,000 \text { platelets } / \mu 1}{9.9 \text { days }} \times \frac{90 \%}{65 \%}
$$

which equaled 35,000 platelets $/ \mu 1$ per day \pm 4300 . The nucleated red cell:megakaryocyte ratio in 15 normal subjects was $465: 1 \pm 60$ and corresponded to a total megakaryocyte number of $6.1 \times 10^{6} / \mathrm{kg} \pm 0.7$. The mean megakaryocyte volume was $4700 \mu^{8} \pm 100,{ }^{7}$ giving a total megakaryocyte mass in normal subjects of $2.8 \times 10^{10} \mu^{3} /$ $\mathrm{kg} \pm 0.3$. The coefficient of variation for platelet turnover and megakayrocyte mass was $\pm 15 \%$ for the group of 15 normal subjects. ${ }^{8}$ In normal marrow $65 \% \pm 3.3$ of the megakaryocytes contained eight nuclei, $23 \% \pm 2.7$ contained 16 nuclei, and $12 \% \pm 1.8$ contained four nuclei. The cell volume per nucleus in normal subjects was 520 $\mu^{3} /$ nucleus \pm 20 . The normal calculated daily platelet production per nuclear unit averaged $49 \pm 5$.

The mean values on these normal subjects were used

\footnotetext{
${ }^{7}$ This value was obtained in Zenker's formaldehyde-fixed sections which produced about $25 \%$ shrinkage in diameter. The volume estimate, therefore, is relative only and does not represent an absolute measurement of megakaryocyte volume in vivo.

${ }^{8}$ Derived from the standard deviation of the individual ratios and on the ratio of the means, which gave comparable values (15).

${ }^{9}$ The mathematical device of expressing platelet production per nuclear unit is employed to reflect the effectiveness of platelet production, and its use is not meant to imply that platelet release occurs continuously throughout the life cycle of a single megakaryocyte.
}

as the normal reference standard to which patient studies were compared.

\section{Thrombocytopenia}

Observations on selected patients with thrombocytopenia are presented in Table II.

\section{Production Disorders}

Hypoproliferation. In patients Nos. 16-22 with hypoproliferative states (marrow hypoplasia and myelophthisis), platelet turnover ranged from $3100-15,200 / \mu 1$ per day, corresponding to a production rate of $0.1-0.4$ of the normal value. The megakaryocyte mass showed a decrease of $0.06-1.7 \mu^{3} \times 10^{10} / \mathrm{kg}$ or $0.02-0.6$ of normal. In one child with aplastic anemia (patient No. 19), the production of platelets increased from 0.4 to 0.8 of normal and megakaryocyte mass from 0.5 to 0.9 of normal under the influence of steroid therapy.

Ineffective production. Patient Nos. 23-30 showed a platelet turnover lower than expected from the megakaryocyte mass, as evidenced by a mean daily platelet production per nuclear unit of 6 (range, 1-13), compared with the normal of $49 \pm 5$. In two patients with vitamin $B_{18}$ deficiency the megakaryocyte mass was increased to four times normal, but platelet turnover was 0.4 of normal, a finding indicating that about $10 \%$ of the expected platelet production occurred from the megakaryocyte mass, i.e., daily platelet production per nuclear unit was 5 and 4 . Similar findings were obtained in patients with DiGuglielmo's syndrome. A mother and daughter (patient Nos. 28 and 29) were of particular interest. Megakaryocyte mass was increased to 1.5 and 2.3 of normal, but the turnover of circulating platelets was reduced to about half of normal giving daily platelet production values less than one-fourth of normal. Another patient (No. 30) had a megakaryocyte mass 5.4 times normal but platelet turnover was only onetenth of normal, indicating that there was only $2 \%$ of the expected platelet production. In all eight patients, the discrepancy between measured platelet turnover and potential platelet production, reflected by the decreased platelet production per nuclear unit, indicated ineffective thrombopoiesis.

\section{Disorders of Distribution}

Splenic enlargement was found to be associated with abnormalities in the intravascular distribution of platelets. In congestive splenomegaly (patient Nos. 31-34), platelet counts varied from 55,000 to $263,000 / \mu l$, platelet life-span ranged from 7.0 to 9.5 days, and immediate recovery of transfused platelets was decreased to 7-23\% as compared with the normal value of $65 \%$. An average immediate recovery of $15 \%$ suggested that as much as 
TABLE II

Thrombocytopenia

\begin{tabular}{|c|c|c|c|c|c|c|c|c|c|c|c|c|}
\hline \multirow[b]{3}{*}{$\begin{array}{l}\text { Subject } \\
\text { No. }\end{array}$} & \multirow[b]{3}{*}{ Diagnosis } & & \multirow{2}{*}{\multicolumn{4}{|c|}{$\frac{\text { Effective thrombopoiesis }}{\text { Platelet }}$}} & \multirow{2}{*}{\multicolumn{4}{|c|}{$\frac{\text { Total thrombopoiesis }}{\text { Megakaryocyte }}$}} & \multirow[b]{3}{*}{$\begin{array}{c}\text { Cell } \\
\text { vol- } \\
\text { ume/ } \\
\text { nucleus }\end{array}$} & \multirow[b]{3}{*}{$\begin{array}{c}\text { Platelet } \\
\text { production/ } \\
\text { nuclear } \\
\text { unit }\end{array}$} \\
\hline & & & & & & & & & & & & \\
\hline & & Count & $\begin{array}{l}\text { Sur- } \\
\text { vival }\end{array}$ & $\begin{array}{l}\mathrm{Re}- \\
\text { covery }\end{array}$ & $\begin{array}{l}\text { Turn- } \\
\text { over }\end{array}$ & $\begin{array}{l}\text { Effec- } \\
\text { tive pro- } \\
\text { duction }\end{array}$ & $\begin{array}{c}\text { Num- } \\
\text { ber }\end{array}$ & Volume* & Mass & $\begin{array}{l}\text { Total } \\
\text { produc- } \\
\text { tion }\end{array}$ & & \\
\hline & & $\mu l$ & days & $\%$ & $\begin{array}{l}\text { platelets/ } \\
\text { ul per } \\
\text { day }\end{array}$ & mal & $\underset{\mathrm{kg}}{\times 10^{\circ} /}$ & $\mu^{2}$ & $\mu^{2} \times 10^{100}$ & Xnor- & $\mu^{3}$ & $\begin{array}{l}\text { platelets/ } \\
\text { NU per } \\
\text { day }\end{array}$ \\
\hline \multicolumn{2}{|c|}{ Mean of $1-15$} & 250,000 & 9.9 & 64.6 & 35,000 & 1.0 & 6.1 & $\mathbf{4 , 7 0 0}$ & 2.8 & 1.0 & 520 & 49 \\
\hline \multicolumn{13}{|c|}{ A. Disorders of Production } \\
\hline \multicolumn{13}{|c|}{ Hypoplasia } \\
\hline 16 & Drug marrow damage & 16,900 & 8.0 & 61.9 & 3,100 & 0.1 & 0.6 & 6,890 & 0.4 & 0.1 & 519 & 30 \\
\hline 17 & Drug marrow damage & $3,800 \pm$ & & 67.0 & & & 0.1 & 6,620 & 0.06 & 0.02 & 495 & \\
\hline 18 & Drug marrow damage & 21,500 & 7.5 & 62.2 & 4,100 & 0.1 & 1.1 & 6,700 & 0.8 & 0.3 & 527 & 20 \\
\hline \multirow[t]{3}{*}{19} & $\begin{array}{l}\text { Congenital steroid respon- } \\
\text { sive marrow hypoplasia }\end{array}$ & & & & & & & & & & & \\
\hline & without steroids & 41,700 & 9.0 & 27.5 & 15,200 & 0.4 & 6.8 & 2,065 & 1.4 & 0.5 & & 42 \\
\hline & $20 \mathrm{mg}$ prednisone/day & 84,000 & 9.0 & 29.4 & 28,600 & 0.8 & 9.8 & 2,570 & 2.5 & 0.9 & & 45 \\
\hline \multirow{2}{*}{20} & Marrow replacement by & & & & & & & & & & & \\
\hline & $\begin{array}{l}\text { fibrosis } \\
\text { Marrow replacement by }\end{array}$ & 15,000 & 5.0 & 27.2 & 9,900 & 0.3 & 2.7 & 6,370 & 1.7 & 0.6 & & 23 \\
\hline 21 & lymphoma & 12,300 & 8.0 & 27.3 & 5,100 & 0.1 & 1.2 & 3,540 & 0.4 & 0.1 & 525 & 50 \\
\hline \multirow[t]{2}{*}{22} & Idiopathic marrow aplasia & $2,400 \ddagger$ & & 68.7 & & & 0.1 & 6,370 & 0.06 & 0.02 & & \\
\hline & Ineffective production & & & & & & & & & & & \\
\hline 23 & $B_{12}$ deficiency & 86,700 & 9.5 & 58.7 & 14,000 & 0.4 & 33.2 & $\mathbf{3 , 4 3 0}$ & 11.4 & 4.1 & 586 & 5 \\
\hline 24 & $\mathrm{~B}_{12}$ deficiency & 72,000 & 8.5 & 75.5 & 10,100 & 0.3 & 26.1 & 4.250 & 11.1 & 4.0 & 547 & 4 \\
\hline 25 & DiGuglielmo's syndrome & 14,600 & 6.5 & 30.8 & 6,600 & 0.2 & $\mathbf{8 4 . 0}$ & 1,410 & 11.8 & 4.2 & 530 & 2 \\
\hline 26 & DiGuglielmo's syndrome & 21,900 & 8.5 & 76.8 & 3,000 & 0.1 & 69.8 & 1,600 & 11.2 & 4.0 & 537 & 1 \\
\hline 27 & DiGuglielmo's syndrome & 56,000 & 6.5 & 42.2 & 18,400 & 0.5 & 18.7 & 3,200 & 6.0 & 2.2 & 546 & 13 \\
\hline 28 & Familial thrombocytopenia & 99,000 & 10.0 & 69.5 & 12,800 & 0.4 & 9.5 & $\mathbf{4 , 4 0 0}$ & 4.2 & 1.5 & 519 & 12 \\
\hline 29 & Familial thrombocytopenia & 86,400 & 8.0 & 54.6 & 17,800 & 0.5 & 15.9 & 4,000 & 6.4 & 2.3 & 498 & 10 \\
\hline \multirow[t]{2}{*}{30} & Idiopathic ineffective & & & & & & & & & & & \\
\hline & thrombopoiesis & 20,300 & 8.0 & 78.3 & 2,900 & 0.1 & 18.6 & 8,180 & 15.2 & 5.4 & 526 & 1 \\
\hline \multicolumn{13}{|c|}{ B. Disorders of Distribution } \\
\hline \multicolumn{13}{|c|}{ Splenomegaly } \\
\hline 31 & Congestive splenomegaly & 263,000 & 9.5 & 23.0 & 108,000 & 3.1 & 19.0 & 4.700 & 8.9 & 3.3 & 507 & 46 \\
\hline 32 & Congestive splenomegaly & 90,600 & 7.0 & 17.0 & 68,500 & 2.0 & 10.2 & 5,890 & 6.0 & 2.1 & 495 & 42 \\
\hline 33 & Congestive splenomegaly & 55,000 & 7.5 & 12.5 & 52,800 & 1.5 & 6.2 & 6,370 & 4.0 & 1.4 & 485 & 48 \\
\hline 34 & Congestive splenomegaly & 55,000 & 9.0 & 7.1 & 77,500 & 2.2 & 8.8 & 6,620 & 5.8 & 2.1 & 509 & 51 \\
\hline 35 & Myelofibrosis & 41,900 & 9.0 & 12.0 & 34,900 & 1.0 & 5.2 & 7,060 & 3.6 & 1.3 & & 38 \\
\hline 36 & Myelofibrosis & & & & & & & & & & & \\
\hline & Presplenectomy & 46,000 & 10.0 & 9.7 & 42,700 & 1.2 & 8.3 & 6,030 & 5.0 & 1.8 & 483 & 31 \\
\hline & Postsplenectomy & 410,000 & 9.5 & 96.2 & 40,400 & 1.2 & 8.8 & 4,190 & 3.7 & 1.3 & 491 & 40 \\
\hline 37 & Myelofibrosis & & & & & & & & & & & \\
\hline & Presplenectomy & 56,700 & 8.5 & 9.6 & 62,500 & 1.8 & 7.7 & 6,290 & 4.9 & 1.7 & & 50 \\
\hline & Postsplenectomy & 394,000 & 9.0 & 78.0 & 50,800 & 1.5 & 8.0 & 4,190 & 3.4 & 1.2 & & 58 \\
\hline 38 & Myelofibrosis & & & & & & & & & & & \\
\hline & Presplenectomy & 68,000 & 9.5 & 12.5 & 51,500 & 1.5 & 8.8 & 5,810 & 5.1 & 1.8 & & 39 \\
\hline & Postsplenectomy & 286,000 & 9.0 & 76.8 & 37,200 & 1.1 & 9.0 & 4,320 & 3.9 & 1.4 & & 37 \\
\hline 39 & Chronic lymphatic leukemia & 152,000 & 8.0 & 20.1 & 85,100 & 2.4 & 11.6 & 5,130 & 6.1 & 2.2 & 507 & 53 \\
\hline 40 & Hodgkin's disease & & & & & & & & & & & \\
\hline & Presplenectomy & 148,000 & 10.0 & 17.0 & 78,400 & 2.2 & 14.7 & 5,200 & 6.9 & 2.6 & 520 & 44 \\
\hline & Postsplenectomy & 856,000 & 10.0 & 99.0 & 77,800 & 2.2 & 17.8 & 3,370 & 6.0 & 2.1 & 533 & 52 \\
\hline C. Disorde & $\begin{array}{l}\text { rs of Destruction } \\
\text { Immune }\end{array}$ & & & & & & & & & & & \\
\hline Idio & opathic thrombocytopenic purp & & & & & & & & & & & \\
\hline 41 & ITP & 16,500 & 0.14 & 57.4 & 185,000 & 5.3 & 17.1 & 7,420 & 12.7 & 4.5 & 510 & 56 \\
\hline 42 & ITP & 5,600 & 0.033 & 64.0 & 239,000 & 6.8 & 23.6 & 8.700 & 20.5 & 7.3 & & 47 \\
\hline 43 & ITP & 32,000 & 0.14 & 67.1 & 307,000 & 8.8 & 34.0 & 6,890 & 24.9 & 8.4 & 505 & 47 \\
\hline 44 & ITP & 17,500 & 0.16 & 71.9 & 137,000 & 3.9 & 18.5 & 7,420 & 13.7 & 4.9 & 547 & 41 \\
\hline
\end{tabular}

* Because of shrinkage due to Zenker's formaldehyde fixation, these values do not represent absolute estimates. Diameter measurements from osmic acid fix 2 d samples were $25 \%$ greater.

‡ Reliable survival measurements not obtained because of isoantibodies resultant from transfusion maintenance. On the basis of megakaryocyte mass present in each patient, platelet survival was estimated to be about 7 days. 
TABLE II-(Continued)

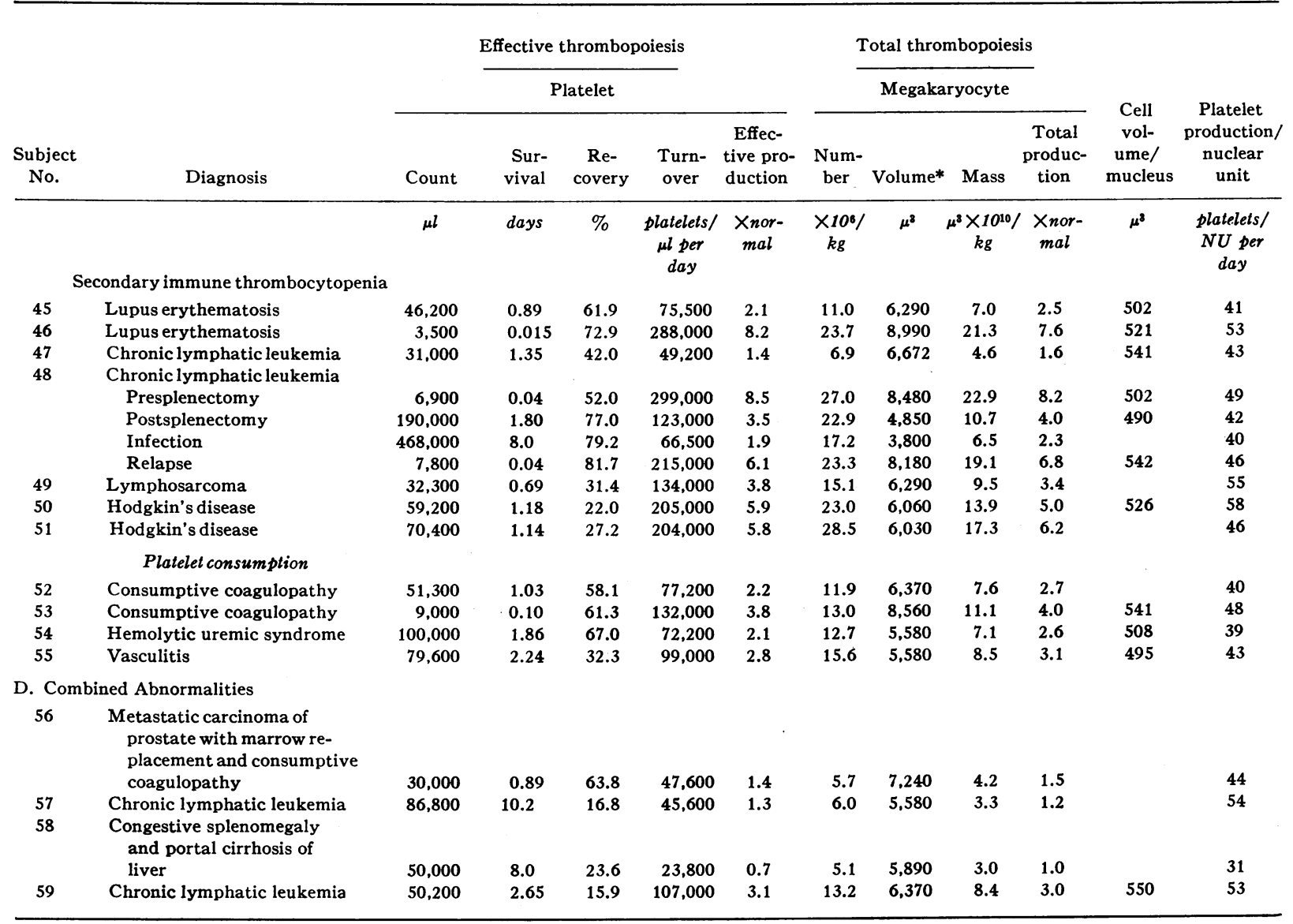

$85 \%$ of the platelets in these patients was sequestered in the spleen. With correction for the sequestered pool, platelet turnover was as much as three times greater than normal in these patients, with corresponding increases in megakaryocyte mass.

In six other patients with marked splenomegaly (patient Nos. 35-38 with myelofibrosis, patient No. 39 with chronic lymphocytic leukemia and patient No. 40 with Hodgkin's disease), there was a low immediate recovery of labeled platelets ranging from 10 to $17 \%$. In these patients the platelet life-span was 8-10 days, and the turnover rate was 1.0-2.4 times normal. The calculation of daily platelet production per nuclear unit for this group was 45 platelets/day \pm 8 , compared with the normal value of $49 \pm 5$, indicating entirely effective thrombopoiesis.

After splenectomy, the immediate platelet recoveries in four patients ranged from 77 to $99 \%$. Furthermore, the rate at which the peripheral platelet count increased postoperatively agreed with the preoperative estimate of platelet turnover (Table III).

\section{Disorders of Destruction}

Immune destruction. In idiopathic thrombocytopenic purpura (ITP) (patient Nos. 41-44) platelet counts ranged between 5600 and $32,000 / \mu 1$, immediate platelet recovery was normal, and platelet life-span was reduced to $48-230 \mathrm{~min}$. Platelet turnover calculations showed

TABLE III

Postsplenectomy Thrombopoiesis

\begin{tabular}{ccc}
\hline $\begin{array}{c}\text { Subject } \\
\text { No. }\end{array}$ & $\begin{array}{c}\text { Platelet turnover } \\
\text { measurement } \\
\text { preoperatively }\end{array}$ & $\begin{array}{c}\text { Daily increase in } \\
\text { platelet count } \\
\text { postsplenectomy* }\end{array}$ \\
\hline & platelet/ & platelet \\
36 & 42,700 & pl per day \\
37 & 62,500 & 43,500 \\
38 & 51,500 & 57,500 \\
40 & 78,400 & 49,300 \\
& & 83,000 \\
\hline
\end{tabular}

* Average of 1 st 4 days postsplenectomy. 
rates of four to nine times normal, and there was a corresponding increase in megakaryocyte mass. Daily platelet production per nuclear unit was normal.

The thrombocytopenia in patient Nos. 45-51 was also considered to have immunologic basis which, however, was secondary to a primary disease (lupus erythematosis, chronic lymphocytic leukemia, or lymphoma). Platelet kinetics in these patients were similar to the ITP group.

Platelet consumption. The thrombokinetics of four patients with platelet consumption (patient Nos. 52-55) were indistinguishable from those with immune thrombocytopenia in respect to the shortening of life-span and parallel increases in platelet turnover and megakaryocyte mass. Production rates were two to four times normal, and thrombopoiesis was effective.

\section{Combined Abnormalities}

The thrombocytopenia of patient Nos. 56-59 was more complex. Patient No. 56 had platelet consumption from intravascular coagulation, but the increase in platelet production to 1.5 times normal was less than

TABLE IV

Thrombocytosis

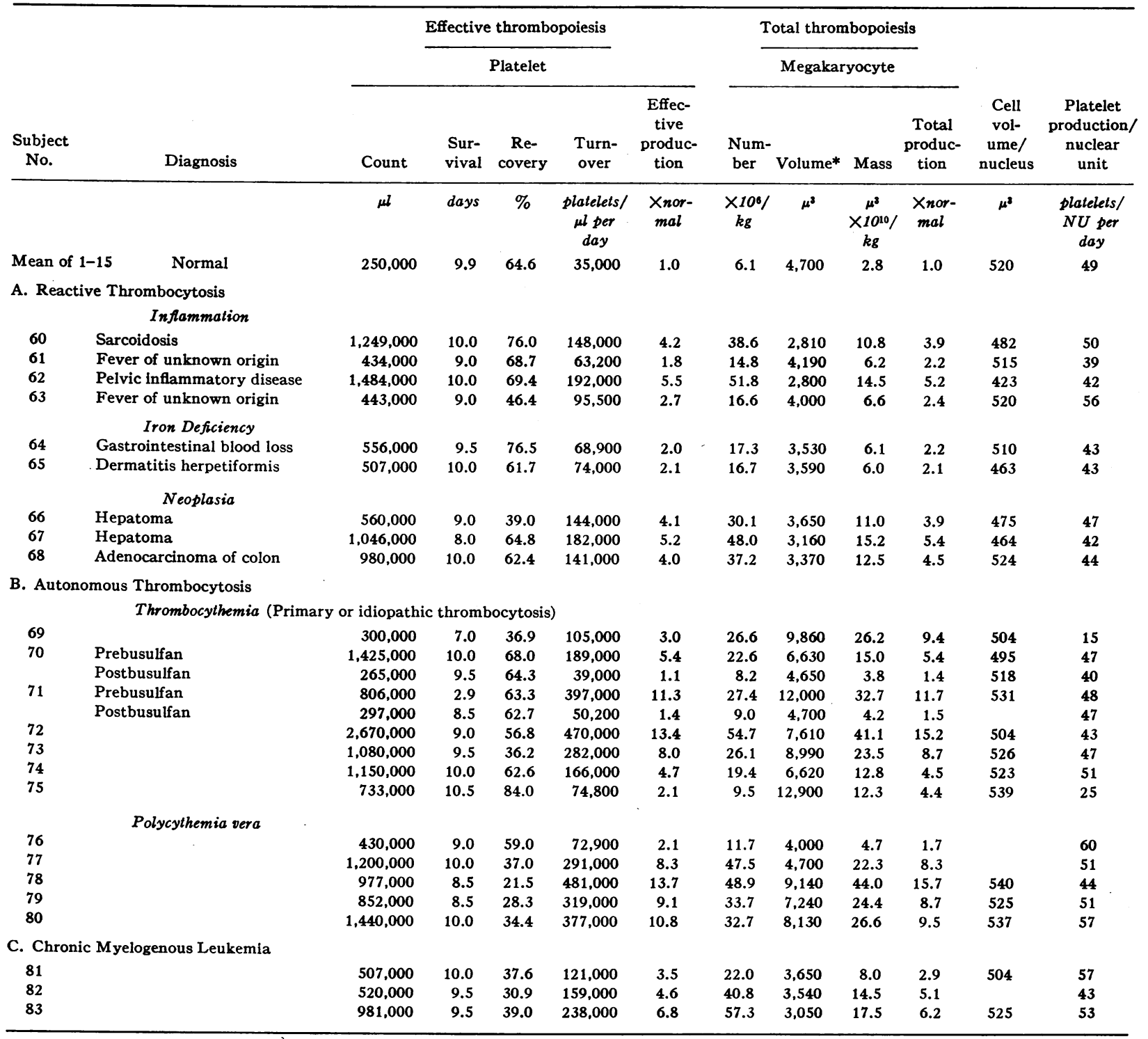

* Because of shrinkage due to Zenker's formaldehyde fixation, these values do not represent absolute estimates. Diameter measurements from osmic acidfixed samples were $25 \%$ greater. 
that of normally responsive marrow for the same degree of thrombocytopenia. In patient No. 57 there was an increase in splenic sequestration without the usual increase in thrombopoiesis presumably as a result of marrow infiltration by neoplastic cells. Patient No. 58 also had increased sequestration and a poor response to thrombocytopenia which may have been related to metabolic liver disease. Patient No. 59, with lymphocytic leukemia, showed increased sequestration due to the infiltrative splenomegaly, a shortened platelet life-span believed to have an immunologic basis, and impaired production due to marrow replacement. These patients illustrate the multiple mechanisms that may operate to produce thrombocytopenia in a single patient.

\section{Thrombocytosis}

Thrombokinetic data in patients with elevated platelet counts are shown in Table IV.

\section{REACTIVE THROMOBOCYTOSIS}

In states of iron deficiency, inflammation or malignant disease arising outside the hematopoietic tissue (patient Nos. 60-68), the platelet count varied from 434,000 to $1,500,000 / \mu 1$. Platelet survival was normal, and platelet turnover varied from 1.8 to 5.5 times normal. Individual mean megakaryocyte volume was uniformly decreased in patients with reactive thrombocytosis. In all instrances, however, the megakaryocyte mass showed increases corresponding to the increased platelet turnover, which indicated that thrombocytosis was achieved by an increase in the number of megakaryocytes rather than by an increase in average megakaryocyte volume.

\section{Autonomous Thrombocytosis}

Patients with primary malignancy of hematopoietic tissue included seven with primary thrombocythemia, five with polycythemia vera, and three with chronic granulocytic leukemia. Platelet levels ranged from 300,000 to $2,700,000 / \mu 1$. With one exception, platelet survival measurements were normal. Thrombocythemia was consistently associated with greater than normal megakaryocyte volume. There was usually agreement between production rates calculated from platelet turnover and from marrow megakaryocyte mass, ranging from 2 to 15 times normal. Patient No. 69 with thrombocythemia, however, appeared to show significant ineffective production. ${ }^{10}$ In patient No. 71 a shortened platelet survival was associated with retinal artery occlusion and multiple small transient sensory and motor signs. The latter signs disappeared after the platelet count, and platelet survival returned to normal on busul-

\footnotetext{
${ }^{10}$ Found to be an outlying observation by the method of Grubbs $(16)(P<0.01)$.
}

fan treatment. The course implied increased platelet consumption in the formation of small vessel obstruction. Patient No. 70 with thrombocythemia was also treated with busulfan, which reduced platelet production to essentially normal levels, and a remission without further therapy was sustained for over a year.

\section{DISCUSSION}

The thrombocyte system is composed of a platelet-producing megakaryocyte compartment and a pool of circulating platelets. Thrombokinetic measurements have been developed to relate the generating mass to the circulating product. The validity of such an approach was demonstrated in this study by independent measurements showing a constant relationship between the marrow megakaryocyte and its output of circulating platelets. Although the platelet release per megakaryocyte is not constant, because of the wide variation found in megakaryocyte volume, the number of platelets formed per megakaryocyte nuclear unit is constant at 49 platelets/ day \pm 5 for 15 normal subjects and $47 \pm 7$ in 67 patient studies. The additional constancy between megakaryocyte volume and nuclear number (Tables II and IV) explains the direct relationship between megakaryocyte mass and platelet turnover (Fig. 4).

An essential step in establishing this relationship relies on using the plasma iron turnover to evaluate the number of immature erythroid cells which is, in turn, related to the megakaryocyte mass through counts of

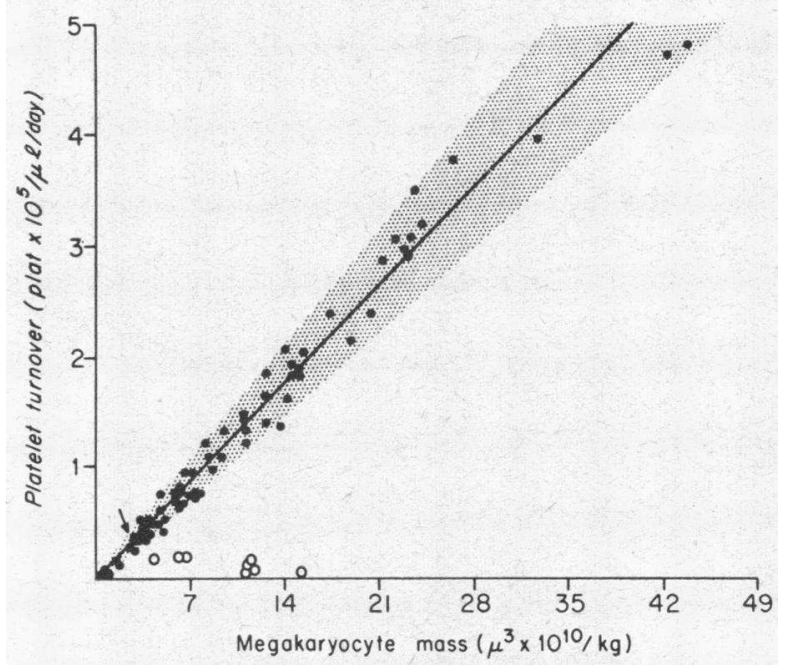

FIGURE 4 The two measurements of platelet production were compared by plotting megakaryocyte mass against platelet turnover. The correlation indicates that these are equivalent measurements. The normal mean value \pm 1 SD is represented by the black square located by the arrow. The $15 \%$ confidence limits $( \pm 1 \mathrm{SD})$ are shown in the shaded area. In eight patients (open circles) marked disparity was seen between platelet turnover and megakaryocyte mass, a finding indicating significant ineffective thrombopoiesis. 
marrow sections. Justification of this use of iron turnover is based on the predominant localization of transferrin-bound radioiron in the erythroid marrow and assumption of a constant relationship between the plasma iron turnover and the marrow normoblasts. It has been shown previously in normal subjects and in a large number of patients that plasma iron turnover measurements usually correlate with the rate of erythropoiesis and erythroid mass $(9,11,12,17)$. While the amount of radioiron localized in the marrow must be known to determine the absolute number of cells, the relative rates of thrombopoiesis as calculated here from erythropoietic rates depend only on the constancy of the per cent of localization of radioiron in the marrow.

The relationship between plasma iron turnover and marrow normoblasts was examined in five subjects (Fig. 2) in whom erythroid number was measured by radioiron dilution technic. The correlation coefficient of 0.99 for erythroid cells calculated by this technic and plasma iron turnover supports the assumption of a constant ratio in radioiron distribution between erythroid and nonerythroid tissues. The relationship can be further examined in states of altered erythropoiesis on the basis of the data presented here. If the data given in this paper are used to estimate megakaryocyte mass from platelet turnover, and then the erythroid: megakaryocyte ratio is applied to calculate the number of nucleated red cells, the latter figure can be compared directly with the plasma iron turnover. Such calculations relating iron turnover to red cell mass in 67 patients gave a correlation coefficient of 0.97 over a red cell production range of less than normal to eight times normal. The two factors which might be expected to interfere with this relationship are a marked decrease in erythropoiesis (where nonerythroid iron removal is increased) and an inadequate supply of iron (where the amount of iron per erythroid cell is reduced). However, extremes were not included in this study. In the eight patients with the lowest plasma iron and the seven patients with the lowest plasma iron turnover values, the correlations were 0.92 and 0.87 , respectively. Thus for the purposes of this study, plasma iron turnover was a satisfactory measure of the number of erythroid precursors.

The effect of platelet pooling in the spleen was important in the determination of platelet turnover. Although the direct determination of splenic pooling was not possible, measurement of the proportion of labeled platelets found in the systemic circulation immediately after infusion (i.e., platelet recovery) provided an indirect but reliable indicator of splenic pool size. Mean recovery of $90 \%$ in six splenectomized patients suggested an immediate loss of about $10 \%$ during the in vitro labeling procedure. The recovery of $65 \%$ in normal sub- jects indicated sequestration of about $25-30 \%$ in the normal spleen. Presumably the recovery of only 10$20 \%$ in patients with splenomegaly reflected splenic pooling of most of the circulating platelets. This assumption was confirmed by measurements of platelet delivery to the circulation after splenectomy, which agreed with the presplenectomy calculations based on sequestration (Table III). Furthermore, the rate of platelet turnover calculated on the basis of splenic pooling matched the measurement of total production based on megakaryocyte mass (Table II).

Alterations in the time required for megakaryocyte development and platelet release might be expected to cause inconsistencies in nuclear production ratios. In stimulated erythropoiesis there is evidence that both the period between cell divisions and the cytoplasmic maturation time may be shortened $(17,18)$. However, in stimulated thrombopoiesis, factors operating to shorten these phases in the megakaryocyte life cycle must be balanced against the time required for the additional mitoses. The persistent correlation between megakaryocyte mass and platelet turnover even in patients with severe thrombocytopenia or thrombocytosis suggests that the duration of the total megakaryocyte life cycle was not greatly altered.

Platelet production in our patients ranged from onetenth of normal to as much as 15 times normal, although levels greater than eight times normal were found only when proliferation was unregulated. Results in the patients with unimpaired marrow function suggested that the normal thrombopoietic system is capable of increasing platelet generation by four to eight times in response to severe thrombocytopenia. These rates are comparable to those previously demonstrated in animals (13).

Alterations in platelet production were accomplished through changes in both the number and the size of megakaryocytes. The number varied over a wide range of 0-14 times normal, whereas volume changes were from one-third to three times normal. Megakaryocyte number and megakaryocyte size usually increased in concert to achieve expanded thrombopoiesis, as in ITP. However these two factors diverged in hypoproliferation (decreased number and increased volume) and reactive thrombocytosis (increased number and decreased volume). When each of these two variables was compared with the platelet count (Fig. 5), no relationship with megakaryocyte number was evident (Fig. 5 A). However there was first-order inverse relationship between megakaryocyte volume and platelet count, the megakaryocytes being proportionately enlarged with thrombocytopenia and diminished in size with thrombocytosis (Fig. $5 \mathrm{~B}$ ). These observations are consistent with previous findings in the experimental animal (13). 


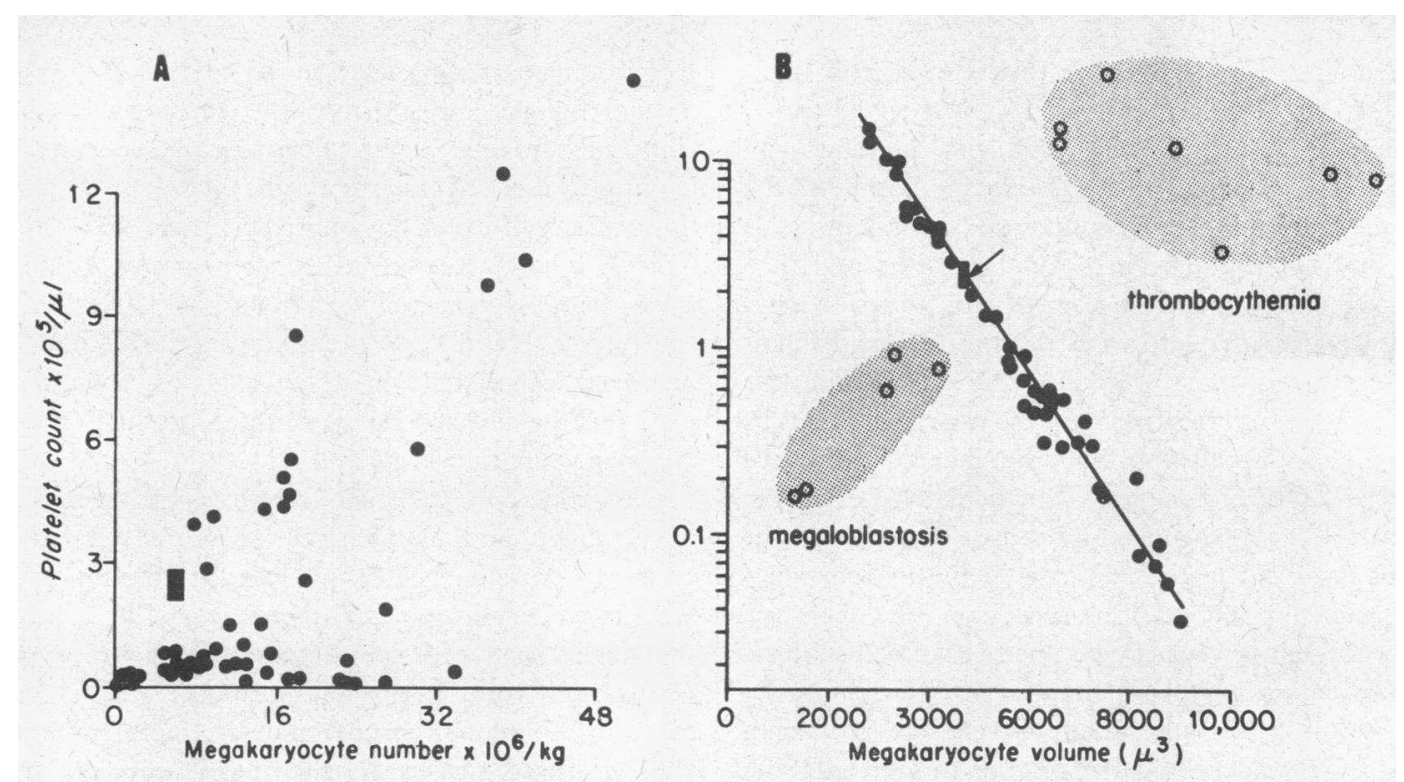

FIGURE 5 The megakaryocyte response to the circulating platelet count is shown in relationship to megakaryocyte number (A) and mean megakaryocyte volume (B). While no correlation was apparent between megakaryocyte number and the platelet count, an inverse first-order relationship was apparent between mean megakaryocyte volume and the circulating platelet count. The normal mean value \pm 1 SD appears as a black rectangle located by the arrow. In two disorders, megaloblastosis and thrombocythemia, the usual relationship between the platelet count and megakaryocyte volume was disturbed. In megaloblastosis the limitation of nuclear replication restricted endomitosis, and hence volume, despite thrombocytopenia. In contrast, thrombocythemia was characterized by increased endomitosis (and therefore volume) in the presence of high platelet counts which is evidence of autonomous proliferation.

Also, the fact that megakaryocyte volume varied directly as the megakaryocyte nuclear number (Fig. 6) showed that in all the patients studied these measurements were equivalent, as illustrated by the relatively constant cell volume per nucleus (Tables II and IV). Thus, megakaryocyte endomitosis appears to be regulated by the requirements for circulating platelets, and either the nuclear lobe count or the megakaryocyte volume may be used as an index of thrombopoietic stimulus.

The course of patient No. 48 illustrates a spectrum of megakaryocyte volume changes in response to alterations in the peripheral platelet count. As the platelet count rose to normal after splenectomy, the megakaryocytes decreased to normal size with relatively little change in their number. When a subphrenic abscess developed, the associated thrombocytosis was accompanied by a decreased megakaryocyte volume (a characteristic finding in the thrombocytosis of infection as shown by patient Nos. 60-63). With the subsequent recurrence of thrombocytopenia, the megakaryocytes again became enlarged. Over the period of observation, the changes in platelet production were largely achieved by changes in megakaryocyte volume, while the number of megakaryocytes remained relatively constant.

The usual inverse relationship between the peripheral platelet count and megakaryocyte volume was not seen in two conditions (Fig. $5 \mathrm{~B}$ ). In one condition, megaloblastosis with thrombocytopenia, both the megakaryocyte volume and the nuclear lobe count were less than normal, a finding consistent with the known impairment of nuclear development in this disorder (14). On

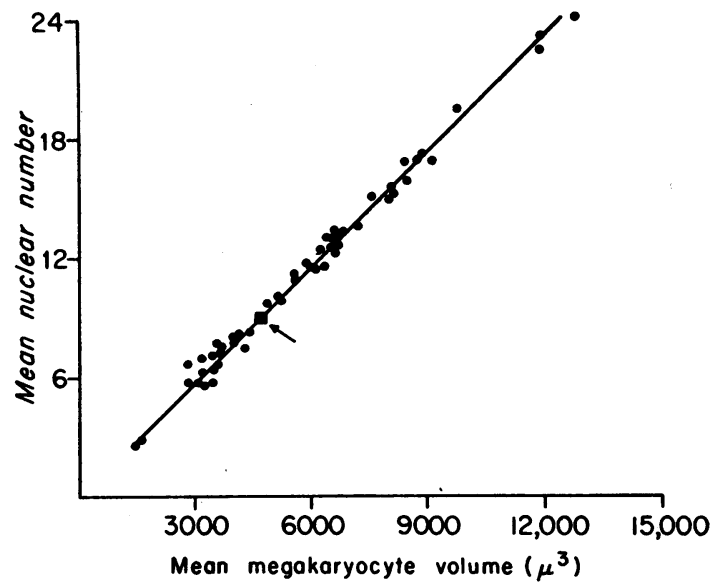

FIGURE 6 A straight-line relationship between the average number of nuclei and the volume of the megakaryocyte indicated that these determinations are equivalent measurements. The normal value $\pm 1 \mathrm{SD}$ is located by the arrow. 
the other hand, megakaryocyte volume and nuclear lobe count were uniformly greater than normal in patients with thrombocythemia (primary thrombocytosis) and in three of the five patients with polycythemia vera. Increased megakaryocyte volume in association with thrombocytosis presumably reflects autonomous marrow behavior in these diseases (19). The fact that megakaryocyte volume in chronic granulocytic leukemia is decreased to an extent appropriate for the degree of thrombocytosis (Table IV) suggests that in this disease platelet production may not be autonomous. Other observers have also reported decreased megakaryocyte size in chronic granulocytic leukemia (20).

Thrombocytopenic disorders. Such disorders may be classified on the basis of disturbances in the production, distribution, or destruction of platelets. The category of hypoproliferation is applied to thrombocytopenia associated with a reduced megakaryocyte mass, as illustrated by a variety of hypoplastic and infiltrative marrow lesions (Table II). Relative hypoproliferation is represented by less than a twofold increase in megakaryocyte mass despite the stimulus of continued thrombocytopenia. The primary defect appears to be impairment of megakaryocyte formation rather than failure of endomitosis.

A second type of disordered production, ineffective thrombopoiesis, is characterized by increased megakaryocyte mass but decreased platelet turnover. This term is applied when daily platelet production per nuclear unit is less than half of that expected. Such disparity between effective and total production was found in vitamin $\mathrm{B}_{1 \mathrm{a}}$ deficiency, DiGuglielmo's syndrome, in a mother and daughter with life-long thrombocytopenia, and as an acquired lesion in another patient. The ineffective thrombopoiesis, described here in five patients with megaloblastic anemia, is similar to the disturbed erythropoiesis in the same condition (21). It is likely that the congenital thrombocytopenia reported by Schulman, Pierce, Lukens, and Currimbhoy (22) and Abildgaard and Simone (23) represents an example of ineffective thrombopoiesis in view of the active megakaryocytopoiesis and probable normal platelet survival described. In the present series, patient No. 30 was considered to have idiopathic ineffective thrombopoiesis resembling patients described by Cohen, Gardner, and Barnett (4), in that a normal platelet survival was associated with active megakaryocyte proliferation.

Distribution abnormalities are known to cause thrombocytopenia; the presence of a splenic platelet pool in normal subjects and the development of thrombocytopenia associated with increased pool size in splenomegaly have been reported by several investigators $(7,24,25)$. The present work confirms the marked increase in platelet sequestration by the enlarged spleen amounting to as much as $90 \%$ or more of the intravascular platelets. In such splenic pooling there is a constant exchange of platelets between systemic and splenic vasculature (7), but the size of the pool remains surprisingly constant under basal conditions (Table III). The observation in animals with induced splenomegaly that platelet turnover was not greatly increased despite the presence of thrombocytopenia $(25,26)$ led to the suggestion that total body platelets, rather than the level of circulating platelets, regulated thrombopoiesis. The present study, however, showed that the platelet turnover and consequently total body platelets were increased two to three times normal in hypersplenic patients and that megakaryocyte mass was correspondingly increased. In patient No. 31 a normal platelet count was achieved despite the enlarged splenic pool by a threefold compensatory increase in platelet production. Furthermore, the average megakaryocyte volume was always increased appropriately for the concentration of circulating platelets, evidencing the thrombopoietic stimulus. Thus on the basis of increased platelet turnover and increased megakaryocyte endomitosis, one can conclude that the regulatory mechanisms are responsive to the blood platelet concentration rather than the total platelet mass.

Disorders of increased platelet destruction have immune or consumptive mechanisms. Current evidence indicates that platelet destruction in ITP, "collagen diseases," and lymphomatous malignancies are mediated by immune mechanisms similar to isoantibody-induced thrombocytopenia (27). The high rates of production in immune thrombocytopenia show that the normal marrow is capable of increasing thrombopoiesis four to eight times normal. No evidence of impaired proliferation or ineffective thrombopoiesis was detected in patients with immune thrombocytopenia.

Platelet consumption occurs during the process of disseminated intravascular coagulation and is accompanied by consumption of fibrinogen, factor V, and factor VIII (28). This disorder is recognized by the shortened platelet survival in association with the characteristic clotting abnormalities. However in some disorders with active vasculitis such as thrombotic thrombocytopenic purpura (29), platelet survival may be shortened without the associated coagulation defects (Table II).

Disorders associated with thrombocytosis. Elevated platelet counts may be designated as reactive or autonomous. Reactive thrombocytosis may occur with inflammation, iron deficiency, or neoplasia not arising from hematopoietic tissue. In this group platelet production was found to be entirely effective at levels of two to four times normal. The highest rates were seen in patient Nos. 60 and 62 with both inflammation and iron deficiency, a finding suggesting the additive effect of each. Megakaryocyte volume was consistently decreased in 
association with the high peripheral platelet count, a finding indicating a decrease in the stimulus for megakaryocyte nuclear reduplication. The marked increase in megakaryocyte number seen in reactive thrombocytosis suggests that factors apparently unrelated to hemostasis influence differentiation of megakaryocytes from the precursor pool.

Autonomous thrombocytosis is the term applied to an increased peripheral platelet count associated with neoplastic proliferation of megakaryocyte endomitosis as characteristically seen in thrombocythemia. Three of our five patients with polycythemia vera had thrombokinetic features suggesting autonomous proliferation of both erythroid and megakaryocytic cell lines. Nuclear lobe counting from marrow aspirates is a practical means of differentiating reactive and autonomous thrombocytosis.

In both normal and pathologic states the similarities between the kinetics of the thrombocyte system and the erythron are remarkable. Both cell types circulate as anuclear forms having a finite life-span within a closed vascular compartment; both elements are generated by precursor marrow cells which proliferate as regulated by mechanisms designed to maintain concentrations in the blood within narrow limits; both are capable of increasing production to about eight times normal; and both have disorders which are related to abnormalities of production and destruction.

\section{ACKNOWLEDGMENTS}

We gratefully acknowledge the technical assistance of Miss Norma Peck, Mrs. Loretta Willis, and Mrs. Barbara Cheney. We also thank Drs. George Marsaglia and James Cook who were mathematical and statistical consultants, and Dr. Eloise Giblett for reviewing the manuscript.

This investigation was supported by U. S. Public Health Service Research Grant 5-R01-HE-06242 and HE 11775-01. A portion of this work was conducted through the Clinical Research Center Facility of the University of Washington supported by the National Institutes of Health (grant FR-37).

\section{REFERENCES}

1. Bull, B. S., M. A. Schneiderman, and G. Brecher. 1965. Platelet counts with the Coulter Counter. Amer. J. Clin. Pathol. 44 : 678.

2. Brecher, G., M. Schneiderman, and E. P. Cronkite. 1953. The reproducibility and constancy of the platelet count. Amer. J. Clin. Pathol. 23: 15.

3. Aster, R. H. 1965. Effect of anticoagulant and $A B O$ incompatibility on recovery of transfused human platelets. Blood. 26: 732 .

4. Cohen, P., F. H. Gardner, and G. O. Barnett. 1961. Reclassification of the thrombocytopenias by the $\mathrm{Cr}^{51}$. labeling method for measuring platelet life span. $N$. Engl. J. Med. 264: 1294.

5. Mills, J. N. 1946. The life-span of the erythrocyte. J. Physiol.Soc. 105: 16P. (Abstr.)
6. Nadler, S. B., J. U. Hidalgo, and T. Bloch. 1962. Prediction of blood volume in normal human adults. Surgery. $51: 224$.

7. Aster, R. H. 1966. Pooling of platelets in the spleen: role in the pathogenesis of "hypersplenic" thrombocytoepenia. J. Clin. Invest. 45: 645.

8. Harker, L. A. 1968. Megakaryocyte quantitation. J. Clin. Invest. 47: 452.

9. Bothwell, T. H., and C. A. Finch. 1962. Iron Metabolism. Little, Brown and Company, Boston.

10. Donohue, D. M., B. W. Gabrio, and C. A. Finch. 1958. Quantitative measurement of hematopoietic cells of the marrow. J. Clin. Invest. 37: 1564.

11. Hosain, F., G. Marsaglia, and C. A. Finch. 1967. Blood ferrokinetics in normal man. J. Clin. Invest. 46: 1.

12. Finch, C. A., K. Deubelbeiss, J. D. Cook, J. W. Eschbach, L. A. Harker, D. D. Funk, G. Marsaglia, R. S. Hillman, S. Slichter, J. W. Adamson, A. Ganzoni, and E. R. Giblett. Ferrokinetics in man. Medicine. In press.

13. Harker, L. A. 1968. Kinetics of thrombopoiesis. J. Clin. Invest. $47: 458$.

14. Japa, J. 1943. A study of the morphology and development of the megakaryocytes. Brit. J. Exp. Pathol. 24: 73.

15. Snedecor, G. W., and W. G. Cochran. 1967. Statistical Methods. Iowa State University Press, Ames. 6th edition.

16. Grubbs, F. E. 1950. Sample criteria for testing outlying observations. Ann. Math. Statist. 21: 27.

17. Hillman, R. S. 1969. The characteristics of marrow production and reticulocyte maturation in normal man in response to anemia. J. Clin. Invest. 48: 454.

18. Hanna, I. R. A., R. G. Tarbutt, and L. F. Lamerton. Shortening of the cell cycle time of erythroid precursors in response to anaemia. Brit. J. Haematol. In press.

19. Adamson, J. W., and C. A. Finch. 1966. Mechanisms of erythroid marrow activation. Trans. Ass. Amer. Physicians Philadelphia. 79: 419.

20. Franzen, S., G. Strenger, and J. Zajicek. 1961. Microplanimetric studies on megakaryocytes in chronic granulocytic leukaemia and polycythaemia vera. Acta Haematol. 26: 182.

21. Finch, C. A., D. H. Coleman, A. G. Motulsky, D. M. Donohue, and R. H. Reiff. 1956. Erythrokinetics in pernicious anemia. Blood. 11: 807.

22. Schulman, I., M. Pierce, A. Lukens, and Z. Currimbhoy. 1960. Studies on thrombopoiesis. I. A factor in normal human plasma required for platelet production; chronic thrombocytopenia due to its deficiency. Blood. 16: 943.

23. Abildgaard, C. F., and J. V. Simone. 1967. Thrombopoiesis. Seminars Hematol. 4: 424.

24. Penny, R., M. C. Rozenberg, and B. G. Firkin. 1966. The splenic platelet pool. Blood. 27: 1 .

25. deGabriele, G., and D. G. Penington. 1967. Regulation of platelet production: "hypersplenism" in the experimental animal. Brit. J. Haematol. 13: 384.

26. Aster, R. H. 1967. Studies of the mechanism of "hypersplenic" thrombocytopenia in rats. J. Lab. Clin. Med. 70: 736.

27. Shulman, N. R., V. J. Marder, and R. S. Weinrach. 1964. Comparison of immunologic and idiopathic thrombocytopenia. Trans. Ass. Amer. Physicians Philadelphia. 77: 65 .

28. McKay, D. G. 1965. Disseminated Intravascular Coagulation: an Intermediary Mechanism of Disease. Harper \& Row, Publishers, New York.

29. Feldman, J. D., M. R. Mardiney, E. R. Unanue, and H. Cutting. 1966. The vascular pathology of thrombotic thrombocytopenic purpura. Lab. Invest. 15: 927. 